\title{
Traitements des mutilés de la Grande Guerre
}

\section{Treatmentsof the mutilated of the Great War}

\section{MOTS-CLEFS : \\ - Première guerre mondiale, blessures maxillo-faciales, prothèse maxillo-faciale. chirurgie plastique}

\section{KEYWORDS:}

- World War I, maxillofacial injuries, maxillofacial prothesis, plastic surgery

$\operatorname{AOS} n^{\circ} 274-2016$

\section{Résumé}

La Première Guerre mondiale, du fait du fort développement de l'artillerie, causa de nombreuses blessures d'un nouveau type : les lésions maxillo-faciales. Ces vastes délabrements sont des plaies béantes à l'aspect si effroyable que de nombreux soldats sont laissés pour morts sur le champ de bataille. Les blessures maxillo-faciales sont caractérisées par d'importantes pertes de substance touchant les maxillaires et les téguments de la face. La reconstruction faciale de ces mutilés, les gueules cassées, nécessite l'invention de nouvelles techniques opératoires et la conception de prothèses innovantes. Le personnel de santé, dont les chirurgiens maxillo-faciaux et les chirurgiens-dentistes, a fait preuve d'une inventivité sans précédent, contribuant ainsi à l'essor considérable de leurs spécialités.
Abstract

The World War I, due to the strong development of the artillery, caused a considerable number of new injuries: the maxillofacial lesions. These large dilapidations are gaping wounds which look so awful that many soldiers are left for dead on the battlefield. The maxillofacial injuries are characterized by significant loss of substance affecting jaws and soft tissues of the face. The facial reconstruction of these disabled ex-servicemen, the "gueules cassées", requires the invention of new surgical techniques and design of innovative prostheses. Health personnel such as maxillofacial surgeons and dentists have shown unprecedented inventiveness, thus contributing to the development of their specialties.

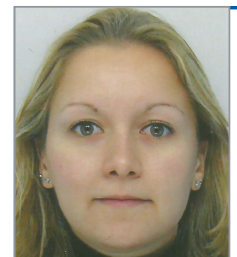

- Virginie ROCHETTE, Docteur en Chirurgie dentaire, CES Biologie buccale, option histo-embryologie, Lauréate de l'Académie Nationale de Chirurgie Dentaire pour l'année 2009.

Jacques MARGERIT, Docteur en Chirurgie dentaire, PU-PH, Service de Prothèse, Faculté d'Odontologie, Université Montpellier 1, 545 av. du Pr. J.-L. Viala, 34193 Montpellier Cedex 5.

\section{INTRODUCTION}

La Grande Guerre a été très meurtrière avec un lourd bilan humain. Contrairement aux conflits précédents où les soldats mouraient de maladie, le pourcentage de morts par blessures de guerre augmente considérablement dès 1914 .

La Première Guerre mondiale marque l'émergence du nouveau type de blessures que sont les atteintes maxillo-faciales.

Les blessés de la face restés défigurés ont été appelés les gueules cassées.
Le nombre et la fréquence de ces lésions ont considérablement augmenté durant la guerre de 1914-1918 pour trois raisons :

Ia durée du conflit (quatre ans et demi de combats),

I la guerre de position avec des tranchées où les hommes sont enterrés face à face, la tête étant ainsi la partie la plus exposée du corps

I la modernisation de l'armement avec un fort développement de l'artillerie comme les obus explosifs et les shrapnells. Les éclats d'obus ont causé à eux seuls plus de $75 \%$ des lésions. 


\section{LES BLESSURES MAXILLO-FACIALES}

Le terme de «blessé maxillo-facial » désignait à l'origine un blessé présentant des lésions au niveau des maxillaires et de la face. Au cours de la guerre de 1914-1918, le sens de ce mot a évolué pour désigner un blessé nécessitant un double traitement à la fois prothétique et chirurgical.

Les blessures rencontrées sont très délabrantes, causées par des obus capables d'arracher des membres, de couper des hommes en deux ou de pulvériser des corps. Au niveau facial, on observe de vastes plaies béantes à l'aspect effroyable (Fig. 1). Les lésions maxillo-faciales sont souvent infectées du fait de leur contact avec les cavités septiques de la face que sont la cavité buccale et les sinus. Ce sont également des blessures souillées soit par la terre, des débris de bois, d'os, de dents ou des morceaux de vêtements entraînés dans la plaie par les projectiles.

Dans la plupart des cas, les blessés présentent des fractures des maxillaires associées à d'importantes pertes de substance osseuses et tégumentaires. Ces soldats nécessitent un nettoyage minutieux des plaies (à renouveler régulièrement) ainsi qu'une immobilisation immédiate des fragments fracturés.

Du fait des retards d'évacuation des gueules cassées (42 jours en moyenne), beaucoup d'hommes vont

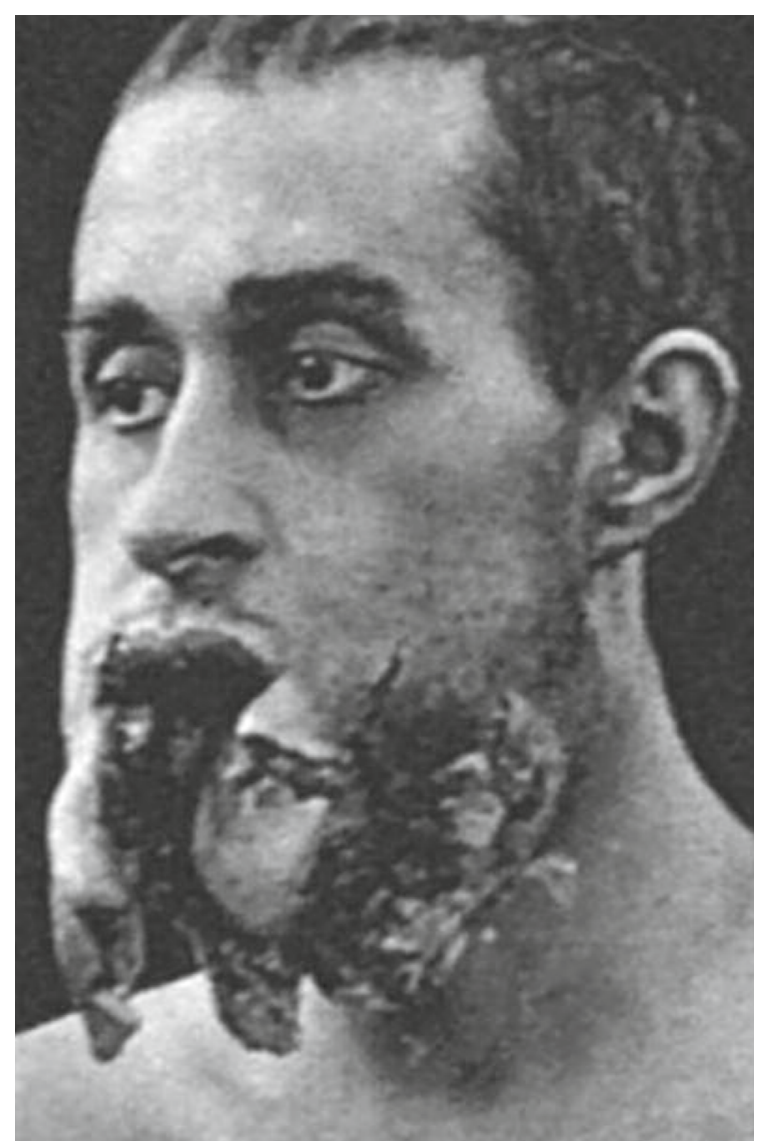

$\triangle$ Fig. 1 : Exemple de lésion maxillo-faciale [3]. souffrir de complications secondaires comme la pseudarthrose (absence de cicatrisation osseuse entre les extrémités fracturées), la consolidation vicieuse (en mauvaise position) des fragments fracturés ou encore la formation de brides fibreuses disgracieuses.

Ces atteintes maxillo-faciales présentent une grande diversité de formes cliniques et une gravité variable en fonction de la nature de l'agent vulnérant, la résistance des tissus lésés, la trajectoire du projectile, ainsi que sa vitesse et sa forme. Chaque blessure maxillo-faciale étant unique, le traitement doit être adapté à chaque patient notamment au niveau prothétique où il faudra pour ainsi dire concevoir et inventer un type de prothèse différent pour chaque blessé. Cela explique les difficultés rencontrées pour les traitements maxillo-faciaux. Le personnel de santé, dont les chirurgiens-dentistes, a donc dû faire preuve d'une grande capacité d'adaptation et d'innovation.

\section{CHIRURGIE MAXILLO-FACIALE}

Jusqu'au début du $\mathrm{XX}^{\mathrm{e}}$ siècle, les opérations de chirurgie maxillo-faciale sont rares et concernent plutôt des atteintes nasales. Devant le nombre considérable de blessés de la face durant la première guerre mondiale, les chirurgiens sont contraints à employer des techniques tombées en désuétude et à en inventer de nouvelles, source des avancées spectaculaires de leur discipline.

\section{Reconstruction d'une perte de substance tégumentaire}

En cas de destruction des parties molles, il est possible de pratiquer des greffes cutanées libres qualifiées d'épidermiques, dermo-épidermiques ou totales en fonction de l'épaisseur du greffon. Devant l'inesthétisme de ces thérapeutiques, les chirurgiens préfèrent les techniques d'autoplasties qui reposent sur l'application de lambeaux en conservant leur pédicule.

Parmi les techniques archaïques, nous pouvons citer la méthode indienne, inventée au II ${ }^{\mathrm{e}}$ siècle, où le lambeau est prélevé au voisinage de la plaie à combler. La greffe italienne, décrite en 1597 par Tagliacozzi, consiste à reconstruire la région nasale grâce à un lambeau réalisé sur l'avant-bras en gardant son pédicule. Le bras reste ainsi immobilisé contre le visage pour conserver la vascularisation du greffon (Fig. 2).

L'autoplastie selon la méthode française repose sur le décollement des tissus entourant la lésion. Les berges de la plaie sont ensuite rapprochées en jouant sur l'élasticité de la peau.

Durant le conflit, H. Gillies met au point une technique innovante héritée de la méthode italienne : les lambeaux en pont ou tubulés. Dans ce cas, le lambeau 


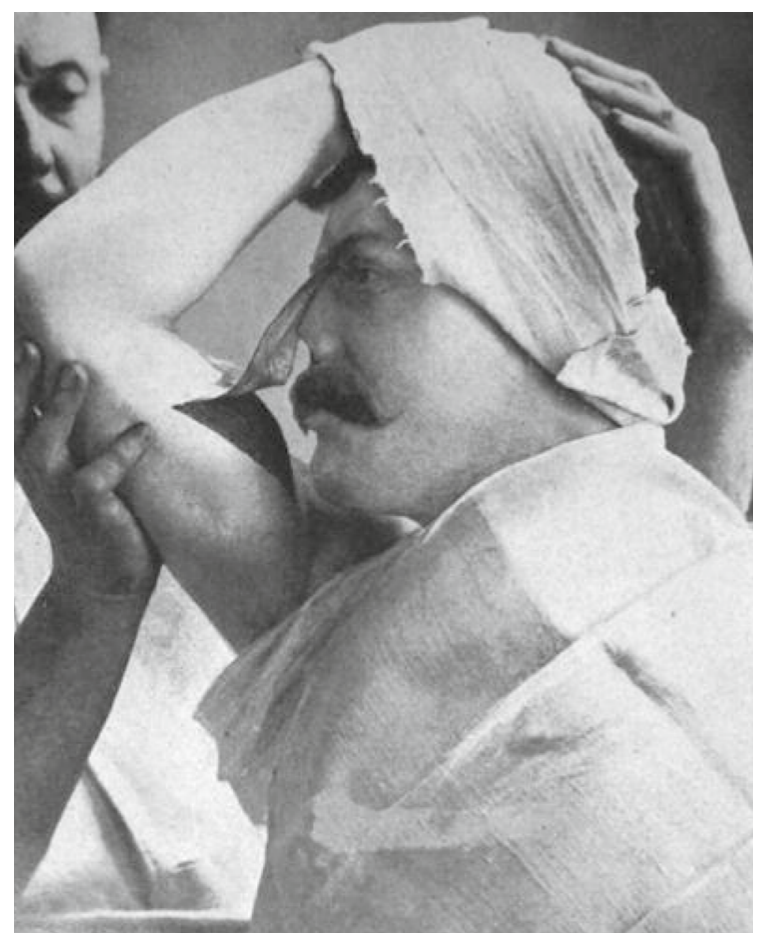

$\triangle$ Fig. 2 : Greffe italienne [9].

est prélevé à distance de la plaie tout en conservant le pédicule. La partie intermédiaire du greffon passe en pont entre la région donneuse et receveuse et peut être suturée pour former un tube (Fig. 3).

L'innovation majeure de la première guerre mondiale est la greffe de Dufourmentel. Celui-ci réalise un lambeau frontal bipédiculé pour reconstituer, après pivotement du greffon, la sangle labio-mentonnière (Fig. 4).

\section{Reconstruction des pertes de substance osseuse}

Face à de vastes fracas osseux, les chirurgiens tentent de combler les pertes de substance grâce à différentes techniques de greffes. En fonction de la nature du greffon, nous distinguons : la greffe osseuse proprement dite (sans périoste), la greffe graisseuse, la greffe cartilagineuse et la greffe ostéo-périostique (méthode de choix).

\section{PROTHĖSE MAXILLO-FACIALE}

Cette discipline connaît un grand essor durant le premier conflit mondial. Les chirurgiens-dentistes conçoivent d'ingénieux appareils pour combler les pertes de substance des maxillaires et résoudre les problèmes de consolidation vicieuse.

Il existe trois grands types de dispositifs :

$\checkmark$ les prothèses immédiates : décrites par Claude Martin dès la fin du XIX ${ }^{e}$ siècle, celles-ci s'interposent directement dans la plaie entre les fragments fracturés. Cette technique a été abandonnée car elle provoquait de nombreuses complications infectieuses ;

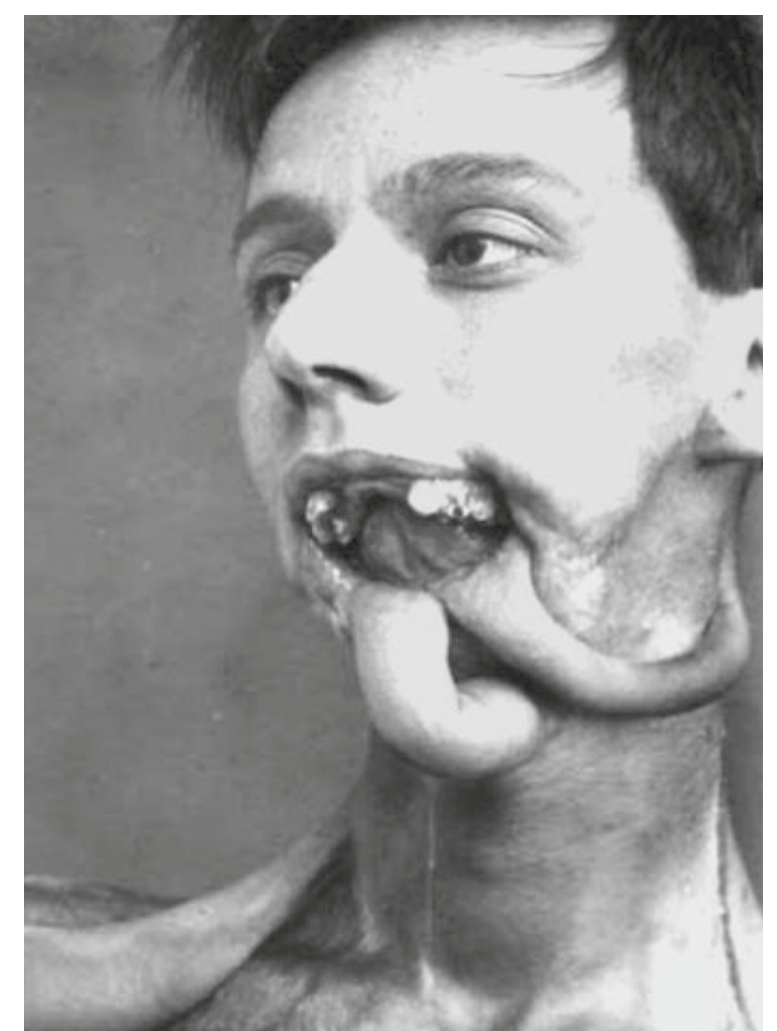

$\triangle$ Fig. 3 : Lambeaux tubulés [9].

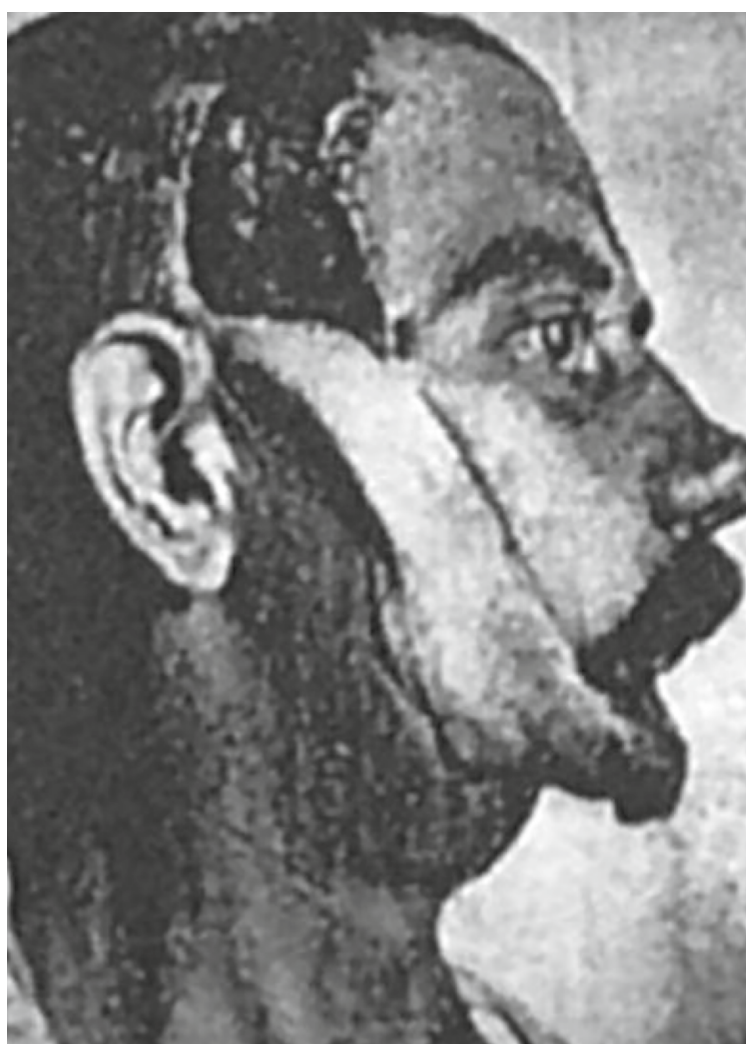

$\triangle$ Fig. 4 : Lambeaux tubulés [9].

$\checkmark$ les prothèses temporaires : utilisées provisoirement durant tout le temps de cicatrisation;

$\checkmark$ les prothèses définitives : en remplacement des précédentes. Elles servent en fin de traitement à pallier les limites de la chirurgie. 


\section{Les prothèses temporaires}

Elles sont divisées en quatre grandes catégories : les prothèses de réduction, les prothèses de contention, les prothèses modelantes et les dispositifs pour dilater les cicatrices.

\section{Prothèses de réduction}

En cas de fracture des maxillaires, la réduction manuelle des fragments devient rapidement impossible (au bout de deux à trois semaines). Les prothèses temporaires de réduction permettent alors de corriger les consolidations vicieuses. Ces appareils sont généralement constitués de gouttières sur lesquelles des forces sont appliquées pour réduire les fractures. On parle de :

Iforce continue si des élastiques ou des ressorts sont utilisés ;

force intermittente si des vis, des écrous ou des vérins sont employés ;

$\checkmark$ force mixte si les deux forces précédentes sont couplées. Ces dispositifs nécessitent un point d'appui qui peut être situé :

I sur un fragment non déplacé du maxillaire fracturé. On parle alors de force monomaxillaire (Fig. 5);

I sur le maxillaire antagoniste. On applique ainsi une force intermaxillaire (Fig. 6) ;

I sur la voûte crânienne. On exerce donc une force cranio-maxillaire ou cranio-mandibulaire (Fig. 7).

\section{Prothèses de contention}

Après la réduction des fractures, des prothèses de contention sont mises en place afin de permettre la cicatrisation en bonne position des fragments fracturés. Celles-ci peuvent être :

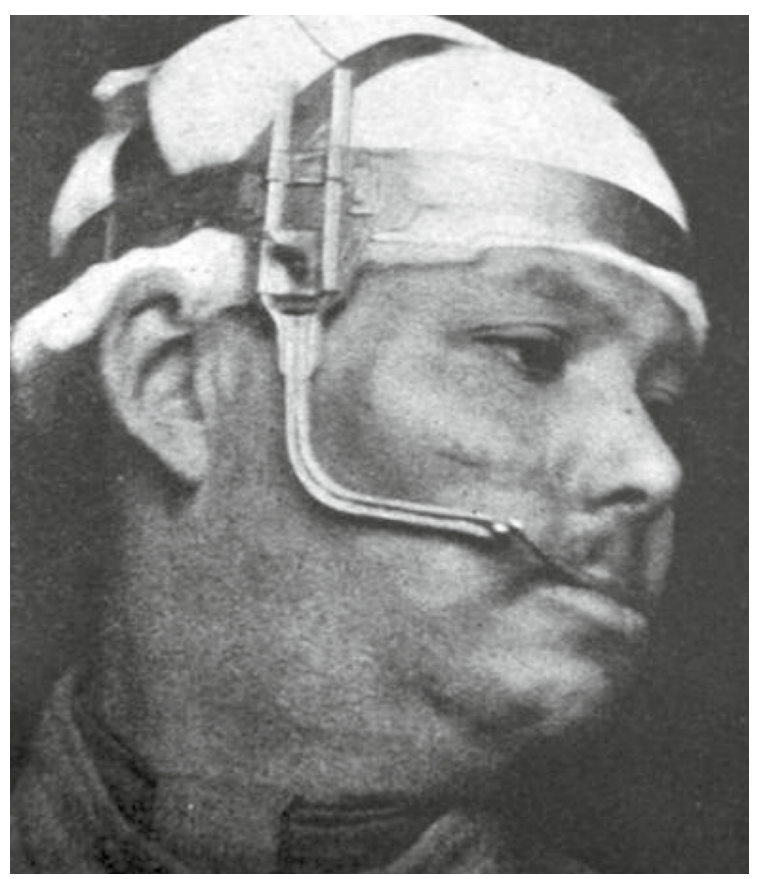

$\triangle$ Fig. 7 : Force cranio-mandibulaire [5].

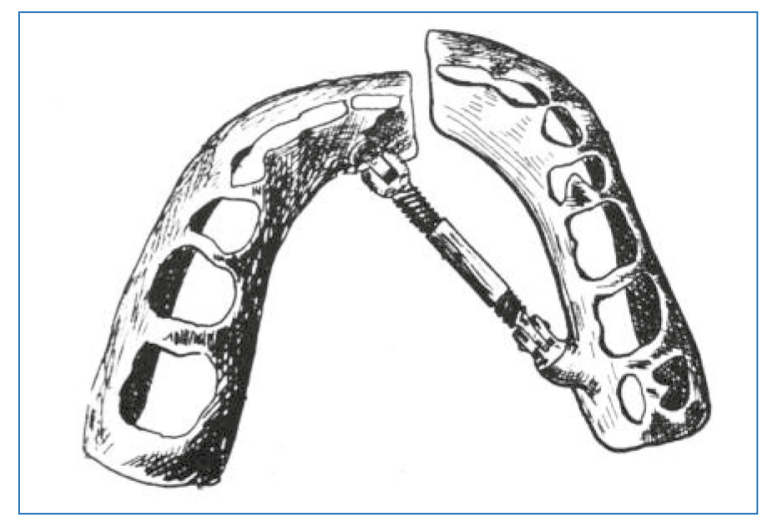

$\triangle$ Fig. 5 : Force monomaxillaire intermittente [5].

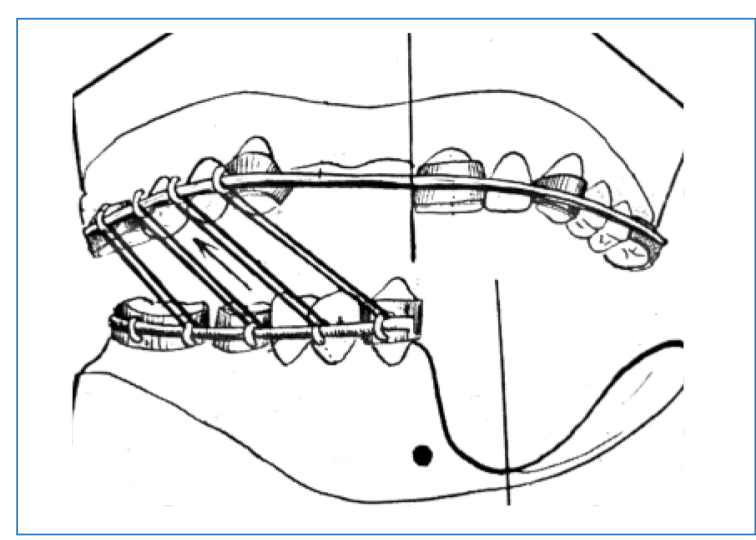

$\triangle$ Fig. 6: Force intermaxillaire continue [5].

I intra-buccales comme des attelles, des ligatures interdentaires, des gouttières de contention, des contentions intermaxillaires (ou blocage des mâchoires) (Fig. 8) ;

I extra-buccales: il s'agit dans ce cas d'une mentonnière reliée à un appui crânien ;

I intra-buccales à point d'appui extra-buccal: une gouttière de contention est reliée à une mentonnière elle-même solidarisée à un appui crânien.

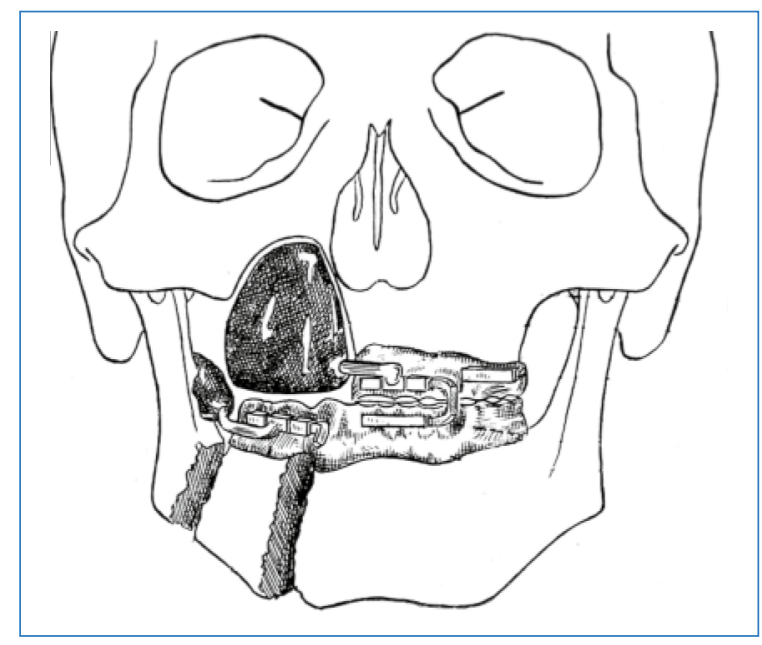

$\triangle$ Fig. 8: Blocage intermaxillaire couplé à une prothèse modelante [10]. 


\section{Prothèses modelantes}

Appelées également prothèses squelettiques pré-opératoires, elles constituent des supports pour les lambeaux d'autoplasties. Il s'agit généralement de blocs de vulcanite fixés sur des gouttières de contention et destinés à combler les pertes de substances osseuses durant les étapes de reconstruction faciale (Fig. 8).

\section{Prothèse pour dilater}

\section{et assouplir les cicatrices}

En cas de rétractions tissulaires, il est possible d'appliquer secondairement une prothèse pour dilater et assouplir les cicatrices. Du point de vue esthétique, ce type d'appareils offre de moins bons résultats qu'une prothèse modelante placée pendant les étapes de reconstruction faciale (Fig. 9).

\section{Les prothèses définitives}

Après la cicatrisation osseuse et des tissus mous, les prothèses temporaires sont remplacées, si nécessaire, par des prothèses définitives.

\section{Extensions de selle prothétique}

Des extensions de selle prothétique sont réalisables lorsque subsistent des pertes de substance alvéolaire non comblées par la chirurgie. Ces dispositifs ressemblent aux prothèses conventionnelles utilisées en pratique courante (Fig. 10).

\section{Prothèses obturatrices}

En cas de perte de substance maxillaire avec une communication bucco-naso-sinusienne, les prothèses obturatrices permettent de restaurer la phonation et

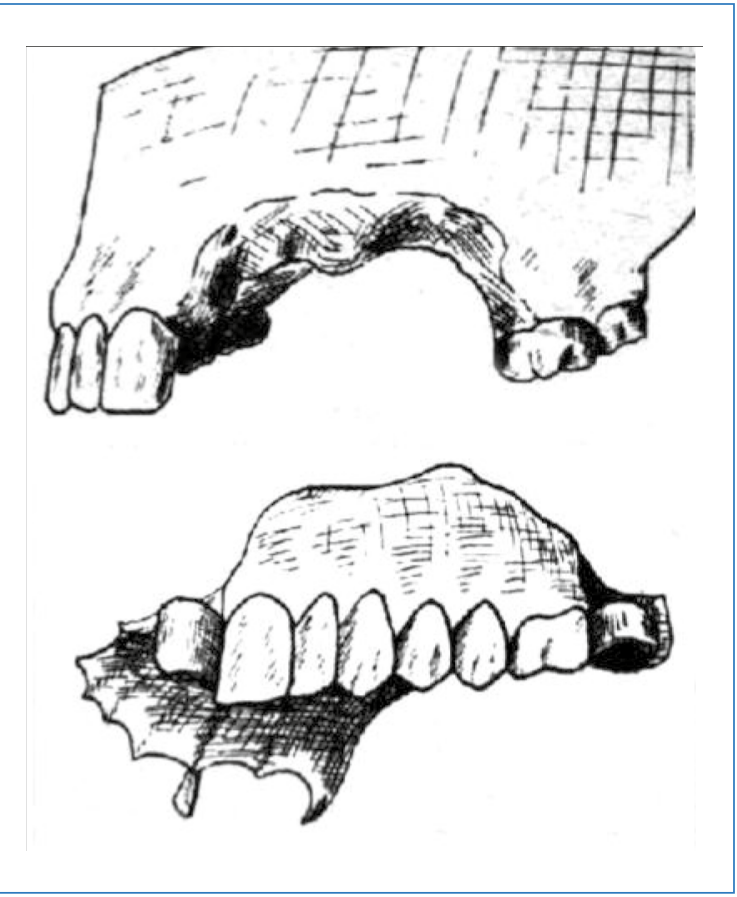

Fig. 10 : Prothèse avec extension de selle [5].

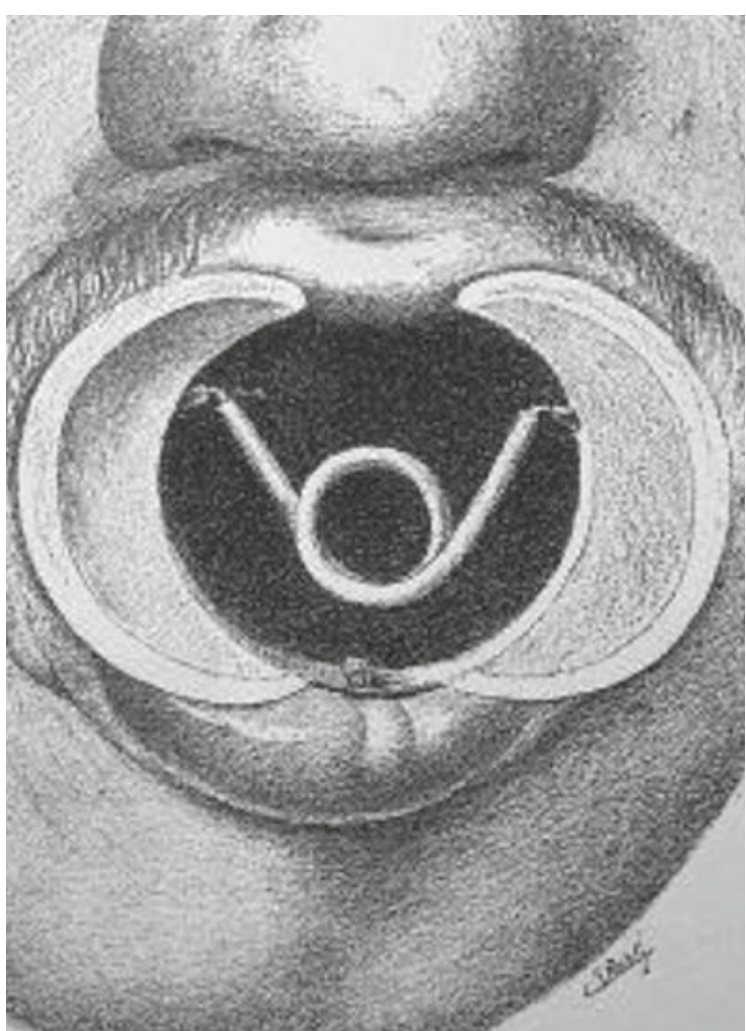

Fig. 9 : Appareil de Duz pour dilater l'orifice buccal [7].

la déglutition. Ces appareils ont dans leur intrados des obturateurs nasaux ou sinusiens. Ces dispositifs agissent par pression (ventouse dans la cavité) ou par frottement (prolongement dans la cavité nasale ou les sinus) (Fig. 11).

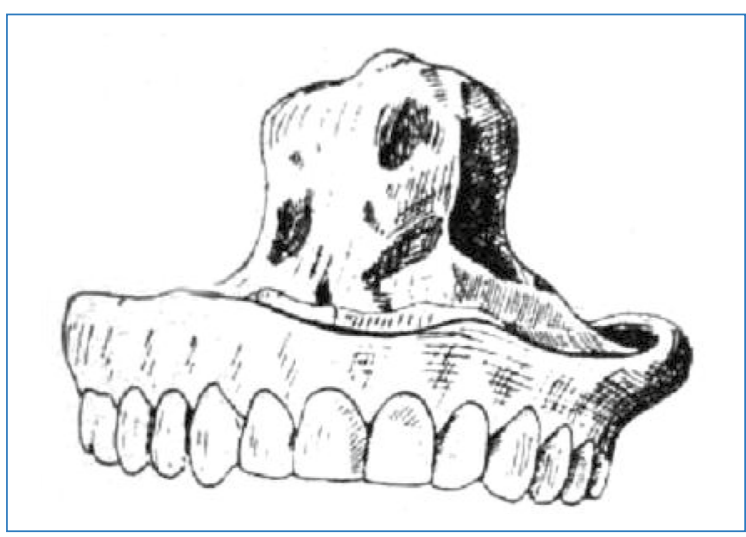

$\triangle$ Fig. 11 : Prothèse obturatrice [5].

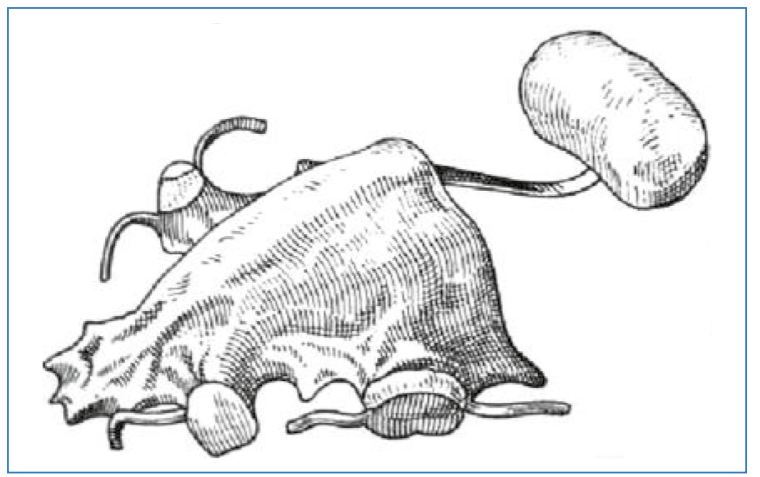

$\triangle$ Fig. 12 : Prothèse vélo-palatine [8]. 


\section{Prothèses vélo-palatines}

Si la perte de substance concerne la région du voile du palais des prothèses vélo-palatines sont alors employées (Fig. 12).

\section{Épithèses}

Les épithèses, ou prothèses faciales, ont pour but de remplacer une partie manquante du visage. Elles sont utilisées quand le massif facial et osseux est trop délabré pour pouvoir être reconstitué par des techniques de chirurgie osseuse et d'autoplasties. Différents types d'épithèses existent en fonction de la zone à reconstituer; citons par exemple les masques faciaux, les prothèses oculo-palpébrales ou les épithèses nasales dont la rétention est souvent assurée grâce à des montures de lunettes (Fig. 13 et 14). Les épithèses sont fabriquées en métal peint, dans des matières plastiques (caoutchouc vulcanisé) ou en gélatine. Ces dispositifs présentent de nombreux inconvénients comme l'altération dans
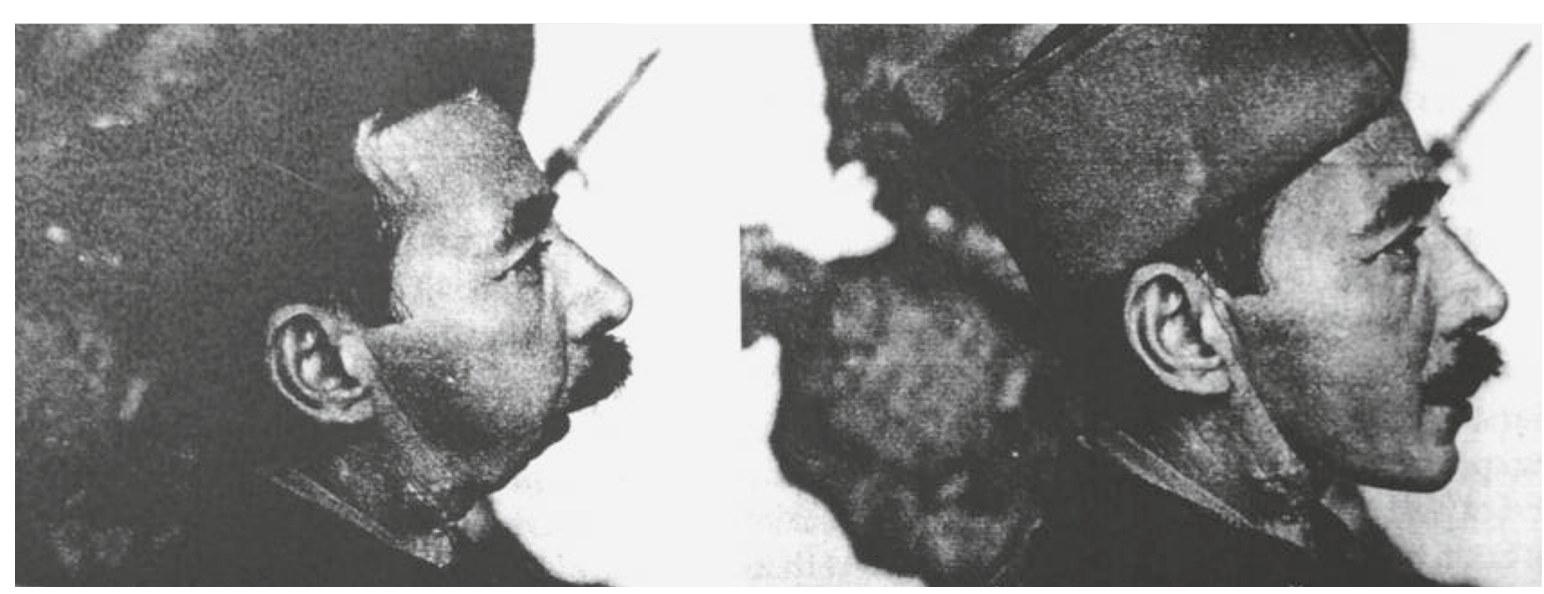

$\triangle$ Fig. 13 : Masque facial [9].
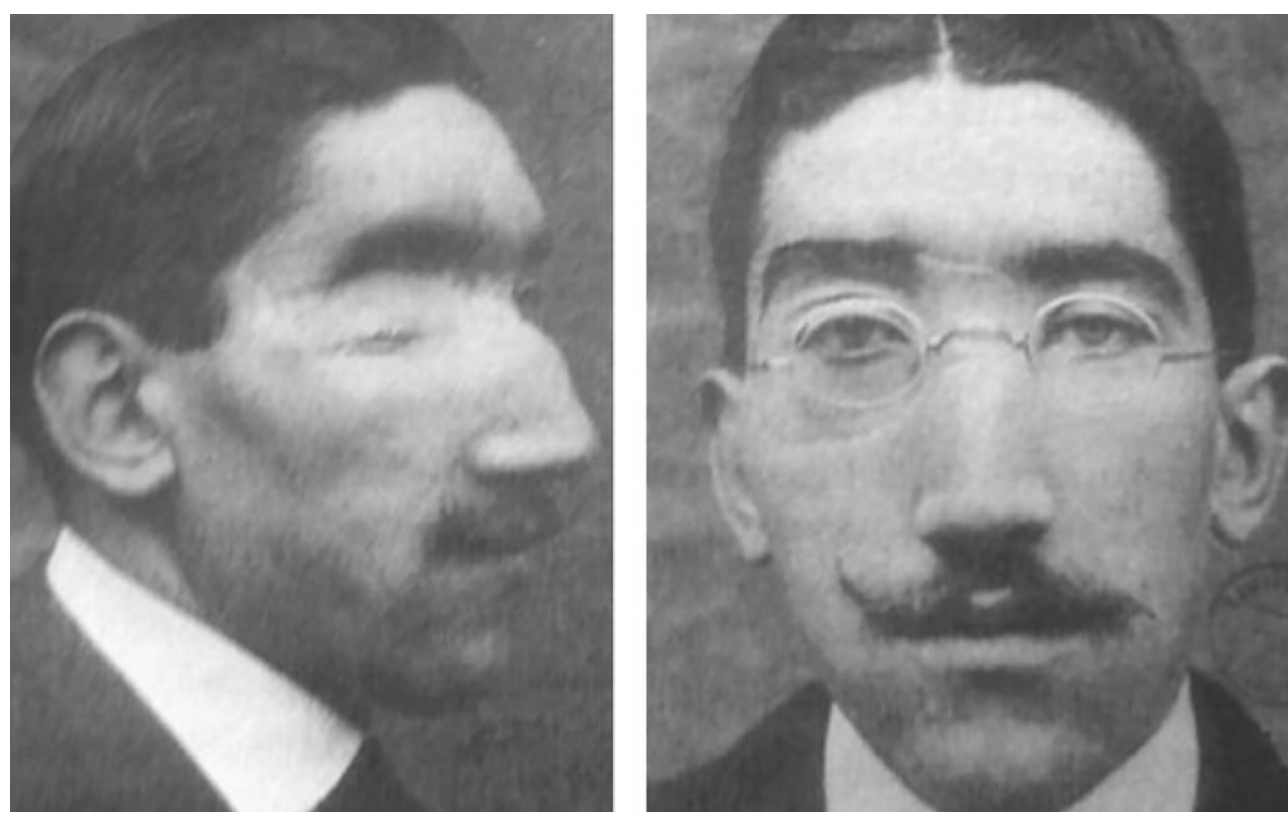

$\triangle$ Fig. 14 : Épithèse oculo-palpébrale [2]. 


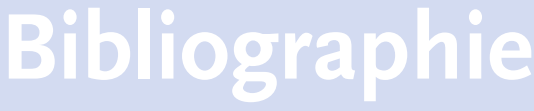

[1] Congrès dentaire interalliés (10 au 13 novembre 1916 : Paris). Paris : Chaix, 1917.

[2] Delaporte S. Gueules cassées de la Grande Guerre. Paris : Agnès Viénot Éditions, 2004, 262 p

[3] Ferret-Dussard K. La chirurgie maxillo-faciale à travers l'histoire : à propos des collections du service de santé des armées du Val-de-Grâce. Paris : Glyphe \& Biotem éd., 2004, 363 p.
[4] Imbert L, Real P. Fractures

de la mâchoire inférieure. Paris : Masson et Cie, 1917, $154 \mathrm{p}$.

[5] Lebedinsky J, Virenque M. Prothèse et chirurgie cranio-maxillo-faciale. Paris : Baillière et fils, $1918,398 \mathrm{p}$

[6] Martinier P, Lemerle G. Prothèse restauratrice bucco-faciale. Paris : Baillière et fils, $1915,360 \mathrm{p}$.

[7] Martinier P, Roy M. La prothèse des maxillaires en chirurgie de guerre. Paris : Baillière et fils, 1917, 93 p.
[8] Ponroy M, Psaume M. Restauration et prothèse maxillo-faciales : fractures, pertes de substance, difformités. Tome VIII. Paris : Masson et Cie, 1950, 540 p.

[9] Rochette V. Prise en charge des blessures maxillo-faciales lors de la Première Guerre Mondiale : Comment reconstruire les visages mutilés ? Th. D. Chir. Dent. : Montpellier : 2009, 305 p.

[10] Virenque M. Chirurgie réparatrice maxillo-faciale : autoplasties, prothèse, restauration. Paris : Maloine et fils, 1940, 253 p. 